\title{
The effect of tracheotomy on drug consumption in patients with acute aneurysmal subarachnoid hemorrhage: an observational study
}

Leiv Arne Rosseland ${ }^{1,2^{*}}$, Jon Narum ${ }^{1}$, Audun Stubhaug ${ }^{1,2}$, Ulf Kongsgaard ${ }^{1,2}$, Wilhelm Sorteberg ${ }^{3}$ and Angelika Sorteberg ${ }^{3}$

\begin{abstract}
Background: Patients with aneurysmal subarachnoid hemorrhage (aSAH) are common in intensive care units (ICU). In patients with aSAH, sedation is used as a neuroprotective measure in order to secure adequate cerebral perfusion pressure (CPP). Compared with the use of an endotracheal tube, a tracheotomy has the advantage of securing the airway at a much lower level of distress, and aSAH patients can often be awakened more rapidly. Little is known about the impact of tracheotomy on the consumption of sedative/analgesic and vasoactive drugs and the maintenance of CPP within defined limits in aSAH patients.

Methods: We conducted an observational study of aSAH patients who underwent percutaneous tracheotomy. A prospective registry of patient data was supplemented with retrospective retrievals from medical records. Sedative, analgesic and vasoactive drug doses were registered for 3 days prior to and after percutaneous tracheotomy, respectively. Blood pressure, CPP, and the mode of mechanical ventilation were registered $24 \mathrm{~h}$ prior to and after tracheotomy.

Results: Between January 2001 and June 2009, 902 aSAH patients were admitted to our hospital; 74 (8\%) were deeply comatose/dying upon arrival. The ruptured aneurysm was repaired in 828 patients (surgical repair 50\%) and percutaneous tracheotomy was performed 182 times in 178 patients (59 men and 119 women). This subpopulation (178 of 828 patients) was significantly older ( 56 vs. 53 years) and presented with a more severe Hunt \& Hess grade $(p<0.001)$. Percutaneous tracheotomy caused a marked decline in mean daily consumption of the analgesics/sedatives fentanyl, midazolam, and propofol, as well as the vasoactive drugs noradrenaline and dopamine. These declines were statistically and clinically significant. The mean CPP was $76 \mathrm{mmHg}$ (SD 8.6) the day before and $79 \mathrm{mmHg}$ (SD 9.6) $24 \mathrm{~h}$ after percutaneous tracheotomy. After percutaneous tracheotomy, mechanical ventilatory support could be reduced to a patient-controlled ventilatory support mode in a significant number of patients $(p<0.001)$.

Conclusions: Percutaneous tracheotomy in aSAH patients is a swift procedure with low risk that is associated with a significant decline in the consumption of sedative/analgesic and vasoactive drugs while clinical surveillance parameters remain stable or improve.
\end{abstract}

Keywords: Tracheotomy, Subarachnoid hemorrhage, Blood pressure, Stroke, Critical care, Midazolam, Fentanyl, Noradrenaline, Dopamine

\footnotetext{
* Correspondence: l.a.rosseland@medisin.uio.no

'Department of Anesthesiology, Division of Emergencies and Critical Care,

Oslo University Hospital - Rikshospitalet, Oslo 0027, Norway

${ }^{2}$ Institute of Clinical Medicine, Faculty of Medicine, University of Oslo, Oslo,

Norway

Full list of author information is available at the end of the article
}

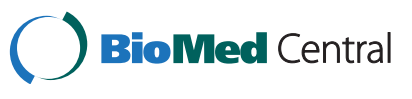

(c) 2015 Rosseland et al.; licensee BioMed Central. This is an Open Access article distributed under the terms of the Creative Commons Attribution License (http://creativecommons.org/licenses/by/4.0), which permits unrestricted use, distribution, and reproduction in any medium, provided the original work is properly credited. The Creative Commons Public Domain Dedication waiver (http://creativecommons.org/publicdomain/zero/1.0/) applies to the data made available in this article, unless otherwise stated. 


\section{Background}

Patients with aneurysmal subarachnoid hemorrhage (aSAH) are common in neurocritical care departments. In conjunction with sedation and continuous monitoring in the intensive care unit (ICU), early aneurysm repair and adequate management of acute hydrocephalus are crucial parts of initial treatment. Local management guidelines include sedation protocols, regulation of arterial blood pressure $(\mathrm{ABP})$, and maintenance of intracranial pressure (ICP) below the defined critical threshold. Adequate cerebral perfusion pressure (CPP), which is defined by the difference between mean $A B P$ and mean ICP, is a cornerstone of optimal management. Following aneurysm repair, our departmental guidelines consider CPP $>70 \mathrm{mmHg}$ to be the goal for most aSAH patients. In the presence of severe cerebral vasospasm, the CPP threshold can be raised to $>90 \mathrm{mmHg}$.

Sedation with opioids, propofol, or benzodiazepines invariably lowers mean $\mathrm{ABP}$, and the administration of vasoactive drugs may be required to maintain the target CPP. Depending on the severity of brain injury, the patient's co-morbidities, and day-to-day assessment of the optimal treatment strategy, the patient may need secure airway access and ventilatory support. While most ICU patients need sedation and pain relief, sedation is also used as a neuroprotective measure in patients with aSAH. When sedated, the brain consumes less oxygen. Furthermore, deeper sedation represents one aspect of preventing or treating increases in ICP.

When the patient regains consciousness he or she may experience discomfort related to the endotracheal tube. When such a stress response is induced, ICP will increase. Frequent coughing may also increase ICP, and can cause unintended excessive cerebrospinal fluid (CSF) drainage through external CSF drains. These situations may necessitate increased sedation. Compared with the use of an endotracheal tube, a tracheotomy has the advantage of securing the airway at a much lower level of distress, and the aSAH patient can often be awakened more rapidly. Little is known about the impact of a tracheotomy on the consumption of sedative/analgesic and vasoactive drugs and the maintenance of CPP within the defined limits. In order to answer these questions, we performed the present observational study on aSAH patients that were managed using a percutaneous tracheotomy (PT).

\section{Methods}

The Regional Committee for Medical and Health Research Ethics for South-East Norway approved the protocol of this study, and the study was exempted from the regulations of informed consent by the Norwegian Directorate of Health. The presentation of the study is prepared according to the STROBE guidelines [1].
All aSAH patients admitted to our hospital between January 2001 and June 2009 were acquired from a prospective registry. Some of these patients represent a sub-group of a previously published analysis in a general ICU population $(\mathrm{N}=1000)$ [2]. The total patient population in our ICU is a mixture of medical and surgical intensive care cases, and the approximate mean number of annual ventilator days during this time period was 4,000. Aneurysmal SAH was the most frequent diagnosis, followed by heart failure, nonaneurysmal intracerebral hemorrhage, and intracerebral or intracerebellar infarction.

Anesthesiologists direct the ICU, and also performed all PTs. The decision of whether and when to establish a PT was based on clinical grounds by the neurosurgeon and the anesthesiologist in charge. Reduced sedation and wake-up were considered once the aneurysm was secured and the patient was stable and well within defined monitoring thresholds. Wake-up and extubation of the orotracheal tube as soon as feasible was one of the primary treatment goals. However, elevated ICP, serious cerebral vasospasm, and/or respiratory problems were indicative of a prolonged time interval before it would be possible to awaken the patient, and hence prompted consideration of tracheotomy. Severely impaired lung function with inspired oxygen fraction $>60 \%$ or positive end expiratory pressure $>10 \mathrm{cmH}_{2} \mathrm{O}$, led to postponement of PT until lung function improved, as would any cerebral instability, in particular increased ICP. The PT was performed by an experienced consultant or by a registrar supervised by a senior consultant in the ICU using a well established technique described elsewhere [3]. A third anesthesiologist secured the airway using direct laryngoscopy and fiber-optic bronchoscopy. The staff reported all PTs to the principal investigator, who consecutively recorded patient demographic data, primary diagnosis, the number of days on mechanical ventilation, duration of the procedure, and complications during the procedure and the following $48 \mathrm{~h}$. Details regarding the aSAH patients were acquired from another registry and included demographic data, Hunt and Hess grade [4] prior to aneurysm repair or prior to sedation, angiographic classification, and mode of aneurysm repair. The sedative/analgesic and vasoactive drug doses were registered during the final 3 days prior to and the first 3 days after PT. Mean ABP, mean ICP, CPP, and the mode of mechanical ventilation were registered the day before and $24 \mathrm{~h}$ after the PT. Mode of mechanical ventilation was graded as $1=$ spontaneous ventilatory support, $2=$ partly controlled ventilation and patient-triggered ventilation, and $3=$ controlled ventilation without patient triggering. The day of PT intervention was excluded from the analyses because the procedure requires variable amounts of analgesics and anesthetics. Each patient's height and weight were measured, reported by relatives, or 
estimated by the intensive care doctor or nurse. The body mass index (BMI) was calculated based on these values. We used 90 -day mortality rates for our analysis. The date of demise originating from the National Population Registry-Norway was collected from the patients' electronic records.

\section{Statistical analysis}

Demographic data, continuous numerical variables, and proportions are presented as mean and standard deviation or standard error, as median and range, or as interquartile range, where appropriate. Proportions were analyzed using the Chi-square test or Fisher's exact test (specified in the results when relevant). The before- and after-effects of the mode of mechanical ventilation were tested statistically using the test for related marginal homogeneity in SPSS $^{\circ}$ version 21 for Windows (Statistical Packages for the Social Sciences), Chicago, IL. Sex differences were tested statistically with an independent sample $t$-test if normally distributed, or a Mann-Whitney $U$ test. Risk factor analyses were performed using a linear regression model or Chi-square test. The statistical tests were performed with $\mathrm{SPSS}^{\circ}$ version 18 for Windows, Chicago, IL. Figures were created with SigmaPlot ${ }^{\circ}$ version 12.5 for Windows (SigmaPlot' Windows, Systat Software, San Jose, CA).

\section{Results}

A total of 902 patients with aSAH were admitted to the hospital during the 8.5-year study period. Seventy-four (8\%) of these patients did not have their aneurysm secured because they were deeply comatose/dying upon arrival. Among the 828 patients that underwent aneurysm repair, PT was performed 182 times in 178 patients (59 men and 119 women, Table 1). Three patients underwent PT twice, and one patient went through a third PT. The mean (SD) duration of mechanical ventilation before PT was 5.3 (2.8) days, and the mean (SD) interval from ictus to PT was 5.8 (4.2) days.

The mean PT procedural time was $12.5 \mathrm{~min}(\mathrm{SD}=8.6$, median $10 \mathrm{~min}$, range $1-50 \mathrm{~min}$ ). There was no procedure-related mortality. Difficulties during insertion of cannula, guide wire, or tracheal tube were registered in 19 patients, and minor bleeding was registered in 11 patients. One procedure was complicated by a short loss of airway control, one patient had perioperative hypoxia in spite of airway control, and one patient developed pneumothorax.

The distributions of sex, age, intervention, preoperative Hunt \& Hess grade, and location of the ruptured aneurysm in patients with and without PT are shown in Table 1. The PT patients were older (mean age 56 years vs. 53 years, $\mathrm{p}=0.005$ ) and presented with more severe Hunt \& Hess grade $(\mathrm{p}<0.001)$. They were in poorer clinical condition prior to aneurysm repair; more than $70 \%$ presented with Hunt \& Hess grade 4 or 5 , compared with $19 \%$ of the non-PT patient population. Patient characteristics among men and women in the PT group are displayed in Table 2. Severity on admission was higher in the female population as $76 \%$ had Hunt \& Hess grade 4 or 5 compared to $70 \%$ in men (Table 1). In the PT population, surgical aneurysm repair was performed in $50 \%$ of patients (Table 1 ). The 90 -day mortality rate was $25 \%$ (95\% CI 18.3 - 31.1).

Mean daily consumption of fentanyl, midazolam, and propofol tended to increase during the 3 days immediately before PT, but decreased significantly during the 3 days immediately after PT (Table 3, Figure 1). Likewise, mean daily consumption of noradrenaline and dopamine dropped significantly after PT and continued to decline during the 3 days after PT (Figure 2). Mean CPP was $76 \mathrm{mmHg}$ (SD 8.6) the day before and $79 \mathrm{mmHg}(\mathrm{SD} 9.6) 24 \mathrm{~h}$ after PT $(\mathrm{p}<0.001$, paired sample

Table 1 Aneurysmal subarachnoid hemorrhage patient characteristics

\begin{tabular}{|c|c|c|c|c|c|c|c|c|c|}
\hline & & Non PT & & & & PT & & & sign \\
\hline Number & & 650 & & & & 178 & & & \\
\hline $\begin{array}{l}\text { Female: Male } \\
\text { (\% female) }\end{array}$ & & $431: 219$ (66.3) & & & & $119: 59(66.9)$ & & & NS \\
\hline $\begin{array}{l}\text { Age } \\
\text { (range in years) }\end{array}$ & & $53(1-87)$ & & & & $56(21-78)$ & & & $p=0.005$ \\
\hline $\begin{array}{l}\text { Surgical aneurysm repair } \\
(\%)\end{array}$ & & 42.9 & & & & 50.0 & & & \\
\hline $\mathrm{HH}$ grade & & $1-3$ & $4-5$ & & & $1-3$ & $4-5$ & & \\
\hline (\%) & & 80.6 & 19.4 & & & 27.0 & 73.0 & & $p<0.001$ \\
\hline $\begin{array}{l}\text { Location of } \\
\text { ruptured aneurysm }\end{array}$ & MCA & $A C A$ & ICA & BA & MCA & ACA & ICA & BA & \\
\hline (\%) & 21.7 & 34.9 & 22.2 & 6.8 & 21.3 & 37.1 & 8.5 & 6.2 & NS \\
\hline
\end{tabular}

PT; percutaneous tracheotomy, $\mathrm{HH}$; clinical grade before securing of the aneurysm/sedation according to Hunt and Hess [4] presented as good grade (HH grade 1-3) and poor grade (HH grade 4 and 5), MCA; Middle cerebral artery, ACA; Anterior Cerebral Aretery, including anterior communicating artery, ICA; Internal carotid artery, BA; basilar artery, sign; statistical significance. 
Table 2 Percutaneous tracheotomy patient characteristics

\begin{tabular}{llll}
\hline & Male & Female & significance \\
\hline Age (years) & $53(33-77)$ & $59(21-78)$ & NS \\
Height $(\mathrm{cm})$ & $181(6.2)$ & $167(4.6)$ & $\mathrm{NS}$ \\
Weight $(\mathrm{kg})$ & $87(15)(60-128)$ & $69(11)(51-115)$ & $\mathrm{NS}$ \\
BMI $\left(\mathrm{kg} / \mathrm{m}^{2}\right)$ & $27(4)$ & $24(3)$ & $\mathrm{NS}$ \\
Baseline HH grade & $4(\mathrm{IQR} 2)$ & $4(\mathrm{IQR} 1.25)$ & $\mathrm{NS}$
\end{tabular}

Demographic data of patients presented as mean (standard deviation), median and range, or inter quartile range (IQR). $\mathrm{BMl}$; body mass index, $\mathrm{HH}$; Hunt Hess grade [4].

$t$-test). Median mechanical ventilator mode was 3 (controlled ventilation) (interquartile range 2-3) before PT and 2 (spontaneous ventilation) (interquartile range $1-3)$ after PT $(\mathrm{p}<0.001$; Figure 3$)$.

\section{Discussion}

The main findings of the present study were that percutaneous tracheotomy (PT) can be performed safely in aSAH patients and that consumption of analgesics, sedatives, and vasoactive drugs became markedly reduced after PT.

\section{Percutaneous tracheotomy}

During the past decade, PT has become a routine method in the ICU. Randomized trials have documented a lower incidence of bleeding and infections compared with open surgical tracheotomy [5-8]. PT is cost-effective [9] and can be performed in a shorter procedure time than surgical tracheotomy [8], with minimal risk of increasing ICP [10]. Our present finding of a median procedural time of $10 \mathrm{~min}$ and few adverse events corroborates these reports. Regarding cost-effectiveness, the present study did not investigate outcome or total hospital time. Nevertheless, the drastic reduction in analgesics/sedatives and vasoactive drugs observed after PT may counterbalance some or all of the cost of performing PT.

Timing of the tracheotomy in ventilated patients has been the focus of some randomized trials [11]. One randomized controlled trial recently reported similar complication rates and patient outcomes in early versus late PT [12]. Because the benefits of timely tracheotomy in the patient population with aSAH may differ from those in the general ICU population, it would have been of interest to perform controlled comparisons in aSAH

Table 3 Administration of sedative drugs before and after PT

\begin{tabular}{llll}
\hline & Before PT & After PT & sign \\
\hline Fentanyl (mg/day) & $3.5(2.7)$ & $2.4(3.9)$ & $\mathrm{p}=0.001$ \\
Midazolam (mg/day) & $277(315)$ & $168(289)$ & $\mathrm{p}<0.001$ \\
Propofol (mg/day) & $1618(1687)$ & $842(1475)$ & $\mathrm{p}<0.001$ \\
\hline
\end{tabular}

Fentanyl, midazolam, and propofol presented as mean (standard deviation) of the three last days before PT and the three days after PT. patients or to analyze this subgroup of patients in the large randomized controlled trials that have already been performed. However, because even the biggest randomized controlled trial addressing the timing of tracheotomy included only a small number of patients with intracranial pathology, a new, prospective, randomized study in this patient population will be necessary.

\section{Vasoactive drugs}

Aneurysmal SAH is a life-threatening event, associated with a mortality rate as high as $75 \%$ in poor grade patients $[13,14]$. Some of this mortality can be attributed to delayed cerebral ischemia. Hence, optimizing the CPP (usually $>70 \mathrm{mmHg}$ ) is a central part of the treatment protocol, and sedated patients most often require vasoactive drugs to obtain the prescribed CPP. The safety of vasoactive drugs regarding the cerebral vasculature is not well documented; therefore, titrating vasoactive drug doses as low as possible is mandatory. This is particularly important regarding the most feared complication of aSAH: cerebral vasospasm. One might raise concerns that the use of vasoactive drugs could exacerbate and/or prolong cerebral vasospasm. However, little is known about the effect of vasoactive drugs on the human intracranial vasculature. One randomized study conducted by Rondeau et al. has compared the combination of noradrenaline and dobutamine with noradrenaline alone [15]. They hypothesized that the dobutamine-induced increase in cardiac output might reduce the incidence of intracranial vasospasm, but failed to show any difference. Experimental data in rabbits indicate that noradrenaline-induced hypertension may increase the diameter and flow in vasospastic intracranial vessels [16]. Our clinical experience supports that noradrenaline is safe, although the benefit of high-dose noradrenaline is controversial.

Phenylephrine represents an alternative to noradrenaline and is commonly used to increase blood pressure to target CPP in neurointensive care [17]. Dopamine is less used [18], but may be indicated if monotherapy with noradrenaline causes bradycardia. Adrenaline should not be used, as it may cause vasospasm [19]. Regardless, the rate of possible vasoactive drug-induced adverse events will be lower if the target $\mathrm{CPP}$ can be maintained at lower levels of vasoactive drugs. The present study demonstrates that PT had a profound impact on vasoactive drug levels, with a marked and ongoing drop in drug usage during the first 3 days after PT.

Because cerebral vasospasm may lead to delayed cerebral ischemia and is a major contributor to poor outcome [20], prophylaxis and treatment of vasospasm are imperative. The neurointensivist will often face the dilemma between optimizing the CPP and minimizing the vasoactive and sedative/analgesic drug doses, especially if the targeted CPP must be increased further owing to 
a

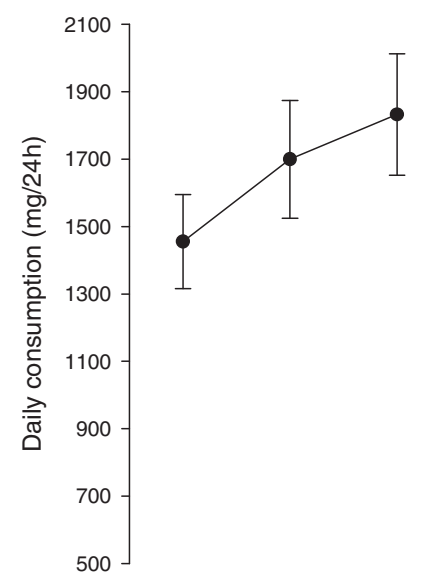

b

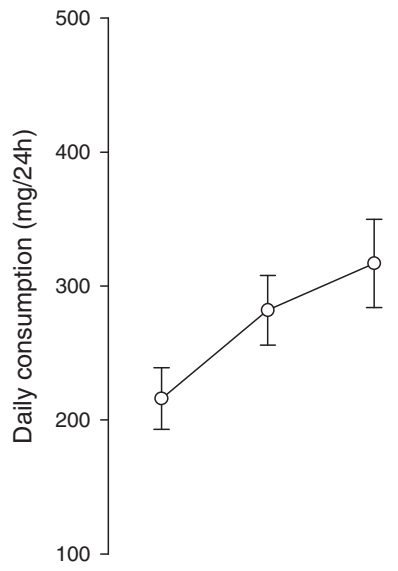

C

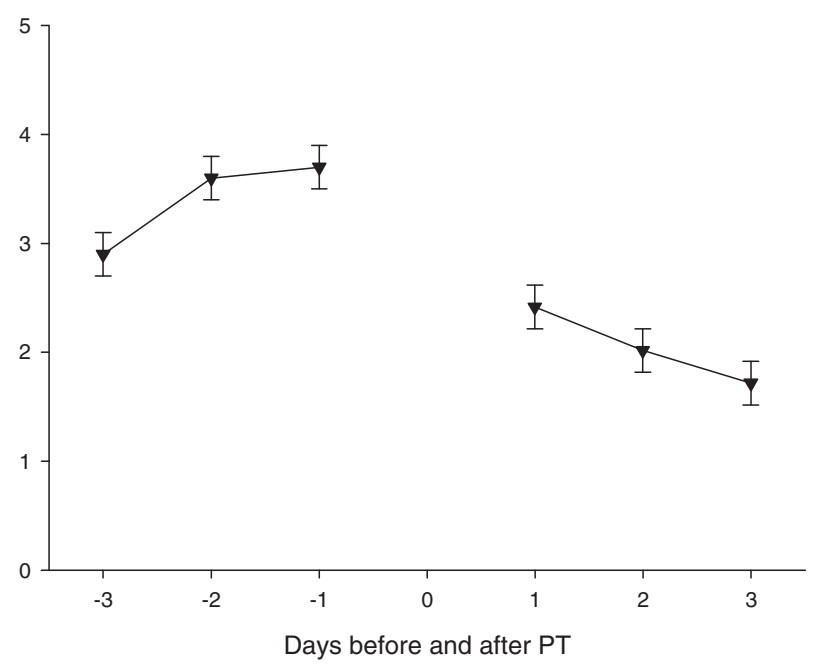

Figure 1 Daily consumption of sedative/analgesic drugs. Daily consumption of fentanyl (a), midazolam (b), and propofol (c) (mg/24 h) presented as mean (SEM) during the 3 days before and the 3 days after tracheotomy. 

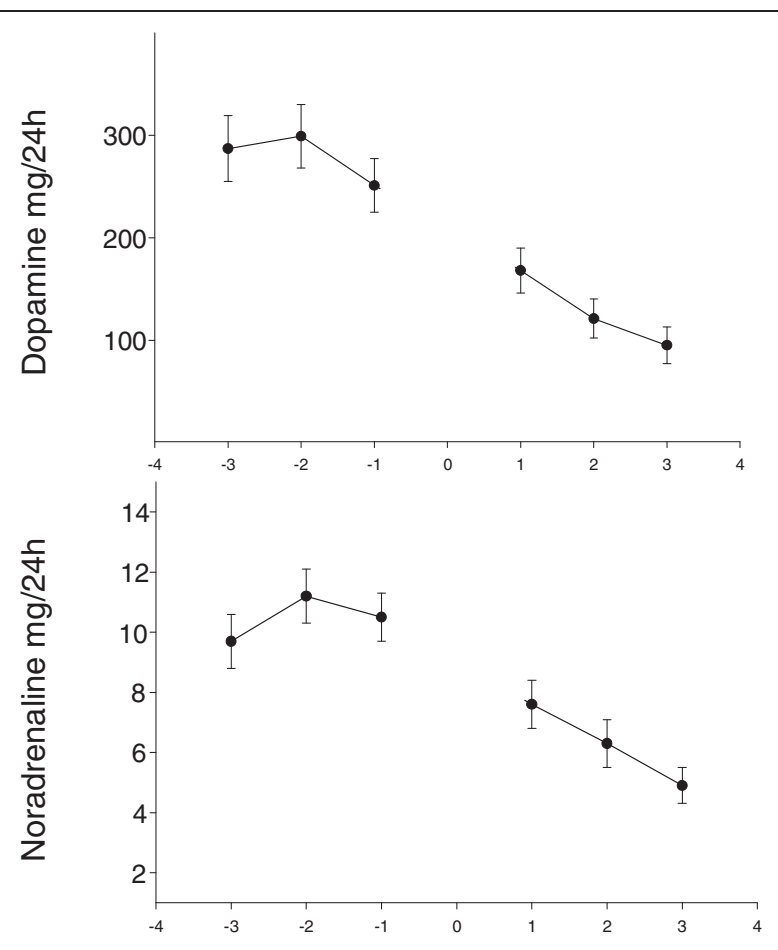

Days before and after PCT

Figure 2 Daily consumption of vasoactive drugs. Daily consumption of dopamine and noradrenaline (mg/24 h) presented as mean (SEM) during the 3 days before and the 3 days after tracheotomy.

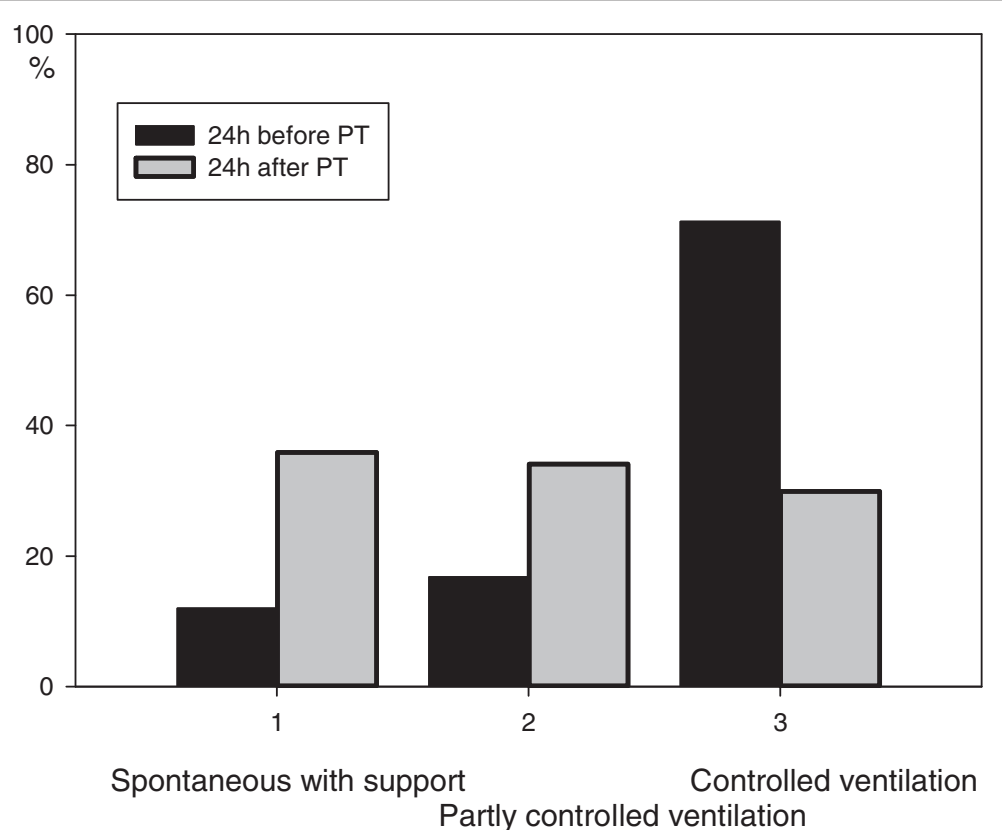

Figure 3 Mechanical ventilator mode $24 \mathrm{~h}$ before and after tracheotomy. The modes of mechanical ventilation were graded as follows: 1 = spontaneous ventilatory support mode; 2 = partly controlled ventilation and patient-triggered ventilation; and $3=$ controlled ventilation without patient triggering. Data were registered $24 \mathrm{~h}$ before (black columns) and $24 \mathrm{~h}$ after (grey columns) tracheotomy. 
documented severe vasospasm. In the present study, after PT, the CPP was easier to control; the mean ICP remained low even when the patient was less sedated. This was likely caused by the reduced level of stress associated with a PT versus an orotracheal tube.

\section{Sedative/analgesic drugs}

A retrospective analysis of 312 patients in a general ICU population documented that administration of sedatives decreased after PT [21], while the observed decrease prior to and after tracheotomy in a 48-h perspective in a study of 1,780 patients did not reach statistical significance [22]. Our study solely comprises aSAH patients, and the fact that these patients require ICP control may have had an impact on our findings. Many aSAH patients experience severely reduced consciousness after the ictus, and prolonged mechanical ventilation is necessary. Sedation continues during invasive ventilatory support, as guided by surveillance thresholds for ICP, CPP, blood oxygenation, and other parameters. We observed a statistically and clinically significant decrease in the consumption of sedative/ analgesic drugs after PT. The doses of fentanyl and midazolam were larger than in a general ICU population [21], and the decrease in drug doses was greater than expected and could not be attributed to the expected natural course of disease. In contrast, the use of sedatives/analgesics was increasing during the 3 days prior to PT. At our ICU, the amount of sedatives used in aSAH patients is usually reduced as soon as is feasible; however, if such a reduction leads to ICP and/or CPP beyond the desired thresholds, sedation is again increased. Hence, tracheotomized patients experience less discomfort when their level of consciousness is increasing, compared with patients having an orotracheal tube. Both discomfort and arousal would contribute to elevations in ICP that in turn would be counteracted with increased sedative/analgesic use. This mechanism, unique for neurointensive patients, in particular those with aSAH, might explain the increasing use of sedatives/analgesics during the days prior to $\mathrm{PT}$, in contrast to a general ICU population [22].

Our study suggests that $\mathrm{PT}$ is particularly well indicated in the aSAH population because it allows ICP and ABP control with much smaller amounts of sedative/analgesic drugs and opioids. This makes it difficult to detect severe vasospasm, and clinical signs are easier to monitor if the patient is awake. Documented serious vasospasm was treated with deep sedation and elevation of the CPP threshold in excess of $90 \mathrm{mmHg}$. No PTs were performed during the phase of serious cerebral vasospasm or other neurological instabilities. After PT, we observed a concomitant increase in CPP that was probably caused by the rapid decrease in sedatives/analgesics, given that there were no changes in the prescribed target CPP values. Although the increase in CPP was statistically significant, it was probably not of clinical impact.

Reduced sedation was associated with changing the ventilator mode; however, a retrospective analysis does not allow conclusions regarding this synergy. The fact that a significant number of patients went from controlled mode to spontaneous ventilation after PT was likely due to the greatly reduced opioid doses.

\section{Population-related factors}

The present 3 -month mortality rate of $25 \%$ was below the average in our general ICU population (30\%) [2]. Mortality was also low compared with previously published data [23,24], particularly considering our high fraction of patients who presented with Hunt \& Hess grades 4 and 5 ("poor grade"). Mortality in this subgroup is reportedly as high as $75 \%[13,14]$. The observation of a significantly poorer clinical grade in the patient population that underwent PT is expected, because patients with an anticipated long course of disease were more likely to be selected for PT. Aneurysmal SAH is more common in women [25,26]; correspondingly, more women underwent PT. The reason for a higher fraction of women being in a poorer clinical state prior to aneurysm repair is not obvious from the available data, but may be related to age, pre-existing co-morbidity, delays in seeking medical attention, larger hemorrhages, or possibly more problems with acute hydrocephalus.

\section{Limitations of the study}

The available observational data cannot answer the question of whether tracheotomy improved outcome or had an effect on the duration of hospitalization in this patient population. Although retrospective analyses may document associations and generate hypotheses, they cannot prove causation. A randomized trial with multifactorial analysis is necessary to investigate these aspects of PT.

Regrettably, the ICU did not register sedation and agitation scores for the patients during the entire study period. This limits our ability to interpret our findings regarding drug consumption. Likewise, GCS could not be retrieved for all days before and after PT in all patients.

\section{Conclusions}

Percutaneous tracheotomy in aSAH patients is a swift procedure with low risk that is associated with a significant decline in the consumption of sedative/analgesic and vasoactive drugs while clinical surveillance parameters remain stable or improve.

\footnotetext{
Abbreviations

aSAH: Aneurysmal subarachnoid hemorrhage; ICU: Intensive care unit; CPP: Cerebral perfusion pressure; ABP: Arterial blood pressure; ICP: Intracranial pressure; CSF: Cerebrospinal fluid; PT: Percutaneous tracheotomy; BMI: Body mass index.
} 


\section{Competing interests}

The authors declare they have no competing interests.

\section{Authors' contributions}

LAR conceived and designed the study, conducted the literature review, collected data, performed analyses, and wrote and critically reviewed the paper. JN participated in study planning, data collection, and analyses, and drafted the manuscript. A Stubhaug conceived and designed the study, conducted the literature review, performed analyses, and wrote and critically reviewed the paper. UK participated in study planning, literature review, and analyses, and reviewed the manuscript. WS conceived and designed the study, conducted the literature review, collected data, performed analyses, and reviewed the paper. A Sorteberg conceived and designed the study, conducted the literature review, collected data, performed analyses, and wrote and critically reviewed the paper. All authors read and approved the final manuscript.

\section{Author details}

${ }^{1}$ Department of Anesthesiology, Division of Emergencies and Critical Care, Oslo University Hospital - Rikshospitalet, Oslo 0027, Norway. ${ }^{2}$ Institute of Clinical Medicine, Faculty of Medicine, University of Oslo, Oslo, Norway. ${ }^{3}$ Department of Neurosurgery, Oslo University Hospital - Rikshospitalet, Oslo, Norway.

Received: 24 September 2014 Accepted: 25 March 2015

Published online: 08 April 2015

\section{References}

1. Von Elm E, Altman DG, Egger M, Pocock SJ, G ${ }^{\circ}$ tzsche PC, Vandenbroucke JP. The Strengthening the Reporting of Observational Studies in Epidemiology (STROBE) statement: guidelines for reporting observational studies. Lancet. 2007;370:1453-7.

2. Rosseland LA, Laake JH, Stubhaug A. Percutaneous dilatational tracheotomy in intensive care unit patients with increased bleeding risk or obesity. A prospective analysis of 1000 procedures. Acta Anaesthesiol Scand. 2011;55:835-41.

3. Kost KM. Endoscopic percutaneous dilatational tracheotomy: a prospective evaluation of 500 consecutive cases. Laryngoscope. 2005;115:1-30.

4. Hunt WE, Hess RM. Surgical risk as related to time of intervention in the repair of intracranial aneurysms. J Neurosurg. 1968;28:14-20.

5. Holdgaard HO, Pedersen J, Jensen RH, Outzen KE, Midtgaard T, Johansen LV, et al. Percutaneous dilatational tracheostomy versus conventional surgical tracheostomy. A clinical randomised study. Acta Anaesthesiol Scand. 1998:42:545-50.

6. Hazard P, Jones C, Benitone J. Comparative clinical trial of standard operative tracheostomy with percutaneous tracheostomy. Crit Care Med. 1991;19:1018-24.

7. Delaney A, Bagshaw S, Nalos M. Percutaneous dilatational tracheostomy versus surgical tracheostomy in critically ill patients: a systematic review and meta-analysis. Crit Care. 2006;10:R55

8. Antonelli M, Michetti V, Di Palma A, Conti G, Pennisi MA, Arcangeli A, et al. Percutaneous translaryngeal versus surgical tracheostomy: A randomized trial with 1-yr double-blind follow-up. Crit Care Med. 2005;33:1015-20.

9. Freeman BD, Isabella K, Cobb JP, Boyle III WA, Schmieg Jr RE, Kolleff MH, et al. A prospective, randomized study comparing percutaneous with surgical tracheostomy in critically ill patients. Crit Care Med. 2001;29:926-30.

10. Kleffmann J, Pahl R, Deinsberger W, Ferbert A, Roth C. Effect of percutaneous tracheostomy on intracerebral pressure and perfusion pressure in patients with acute cerebral dysfunction (TIP Trial): an observational study. Neurocrit Care. 2012;17:85-9.

11. Griffiths J, Barber VS, Morgan L, Young JD. Systematic review and meta-analysis of studies of the timing of tracheostomy in adult patients undergoing artificial ventilation. BMJ. 2005;330:1243.

12. Young D, Harrison DA, Cuthbertson BH, Rowan K. Effect of early vs late tracheostomy placement on survival in patients receiving mechanical ventilation: the TracMan randomized trial. JAMA. 2013;309:2121-9.

13. Le Roux PD, Elliott JP, Newell DW, Grady MS, Winn HR. Predicting outcome in poor-grade patients with subarachnoid hemorrhage: a retrospective review of 159 aggressively managed cases. J Neurosurg. 1996;85:39-49.
14. Mocco J, Ransom ER, Komotar RJ, Schmidt JM, Sciacca RR, Mayer SA, et al. Preoperative prediction of long-term outcome in poor-grade aneurysmal subarachnoid hemorrhage. Neurosurgery. 2006;59:529-38.

15. Rondeau N, Cinotti R, Rozec B, Roquilly A, Floch H, Groleau N, et al. Dobutamine-induced high cardiac index did not prevent vasospasm in subarachnoid hemorrhage patients: a randomized controlled pilot study. Neurocrit Care. 2012;17:183-90.

16. Neuschmelting V, Fathi AR, Hidalgo Staub ET, Marbacher S, Schroth G, Takala J, et al. Norepinephrine-induced hypertension dilates vasospastic basilar artery after subarachnoid haemorrhage in rabbits. Acta Neurochir (Wien). 2009;151:487-93.

17. Meyer R, Deem S, Yanez ND, Souter M, Lam A, Treggiari MM. Current practices of triple-H prophylaxis and therapy in patients with subarachnoid hemorrhage. Neurocrit Care. 2011;14:24-36.

18. Audibert G, Pottie JC, Hummer M, Torrens J. Anesthesia and intensive care of subarachnoid hemorrhage. A survey on practice in 32 centres. Ann Fr Anesth Reanim. 1996;15:338-41.

19. Palma JA, Fontes-Villalba A, Irimia P, Garcia-Eulate R, Martinez-Vila E. Reversible cerebral vasoconstriction syndrome induced by adrenaline. Cephalalgia. 2012;32:500-4.

20. Awad IA, Carter LP, Spetzler RF, Medina M, Williams FC. Clinical vasospasm after subarachnoid hemorrhage: response to hypervolemic hemodilution and arterial hypertension. Stroke. 1987;18:365-72.

21. Nieszkowska A, Combes A, Luyt CE, Ksibi H, Trouillet JL, Gibert C, et al. Impact of tracheotomy on sedative administration, sedation level, and comfort of mechanically ventilated intensive care unit patients. Crit Care Med. 2005;33:2527-33.

22. Veelo D, Dongelmans D, Binnekade J, Korevaar J, Vroom M, Schultz M. Tracheotomy does not affect reducing sedation requirements of patients in intensive care - a retrospective study. Crit Care. 2006;10:R99.

23. Nieuwkamp DJ, Algra A, Blomqvist P, Adami J, Buskens E, Koffijberg H, et al. Excess Mortality and Cardiovascular Events in Patients Surviving Subarachnoid Hemorrhage: A Nationwide Study in Sweden. Stroke. 2011;42:902-7.

24. Samuels O, Webb A, Culler S, Martin K, Barrow D. Impact of a dedicated neurocritical care team in treating patients with aneurysmal subarachnoid hemorrhage. Neurocrit Care. 2011;14:334-40.

25. Lindekleiv H, Sandvei MS, Njolstad I, Lochen ML, Romundstad PR, Vatten L, et al. Sex differences in risk factors for aneurysmal subarachnoid hemorrhage: a cohort study. Neurology. 2011;76:637-43.

26. Sandvei MS, Lindekleiv $H$, Romundstad PR, Muller TB, Vatten $L$, Ingebrigtsen T, et al. Risk factors for aneurysmal subarachnoid hemorrhage - BMI and serum lipids: 11-year follow-up of the HUNT and the Tromso Study in Norway. Acta Neurol Scand. 2012;125:382-8.

\section{Submit your next manuscript to BioMed Central and take full advantage of:}

- Convenient online submission

- Thorough peer review

- No space constraints or color figure charges

- Immediate publication on acceptance

- Inclusion in PubMed, CAS, Scopus and Google Scholar

- Research which is freely available for redistribution 